\title{
Validation of the shortened modified look locker inversion recovery (Sh-MOLLI) sequence for cardiac gated TI mapping
} Stefan K Piechnik*, Vanessa M Ferreira, E Dall'Armellina, Stefan Neubauer and Matthew D Robson

Address: University of Oxford, Oxford, UK

* Corresponding author

from 13th Annual SCMR Scientific Sessions

Phoenix, AZ, USA. $21-24$ January 2010

Published: 21 January 2010

Journal of Cardiovascular Magnetic Resonance 20 I0, I2(Suppl I):O70 doi:I0.1 I86/I532-429X-I2-SI-O70

This abstract is available from: http://jcmr-online.com/content/I2/SI/O70

(c) 2010 Piechnik et al; licensee BioMed Central Ltd.

\section{Background}

T1-mapping of the myocardium can potentially detect, quantify and monitor subtle diffuse pathology without the use of contrast agent. One proven method is the MOLLI (Modified Look-Locker Inversion-recovery) technique, offering single-slice T1-mapping based on three sequential inversion-recovery (IR)-prepared experiments in a 17-heartbeat breath-hold. However, long breathholds can limit clinical application in patients. We propose and test a novel variant (Sh-MOLLI) that is approximately two times faster.

\section{Materials and methods}

10 normal volunteers ( 7 males; age $35 \pm 7$ years) underwent CMR imaging at $1.5 \mathrm{~T}$ (SIEMENS, Avanto) using MOLLI [Messroghli. JMRI. 2007:26(4):1081-6] and ShMOLLI. Basal, mid-cavity and apical short-axis T1 maps were obtained. Sh-MOLLI was implemented as 3 IR experiments split over 9 heartbeats to collect $5+1+1$ images at $\mathrm{TI}=100+[0,1,2,3,4]^{*}$ Heartbeat_Interval, $180,360 \mathrm{~ms}$. IRs were separated by only one heartbeat. Typical SSFP readout parameters: $\mathrm{TE}=1.1 \mathrm{~ms}, \mathrm{TR}=206 \mathrm{~ms}$, flip angle $=$ $35^{\circ}, \mathrm{FOV}=340 \times 116 \mathrm{~mm}$, matrix $192 \times 116$, interpolated voxel size $0.9 \times 0.9 \times 8 \mathrm{~mm}$. Sh-MOLLI samples from the second and third IR are taken into account only if T1 estimate is shorter than the heartbeat interval, and they improve standard error of fit. Nonlinear fitting was implemented in $\mathrm{C}++$ directly in the image reconstruction pipeline utilizing parallel processing with T1 maps available for viewing on the console immediately after acquisition.
Offline post-processing involved manual tracing of endoand epi-cardial contours to calculate $\mathrm{T} 1$ statistics in myocardial segments 1 to 16 of the AHA 17-segment model.

\section{Results}

T1-maps produced by either method did not differ visually. T1-estimates were similar in all but 3 segments (Fig. 1). Across all 160 segments pooled together, Sh-MOLLI T1-estimates were lower by $10.2 \pm 24$ ms (i.e. $-1 \%$, p <

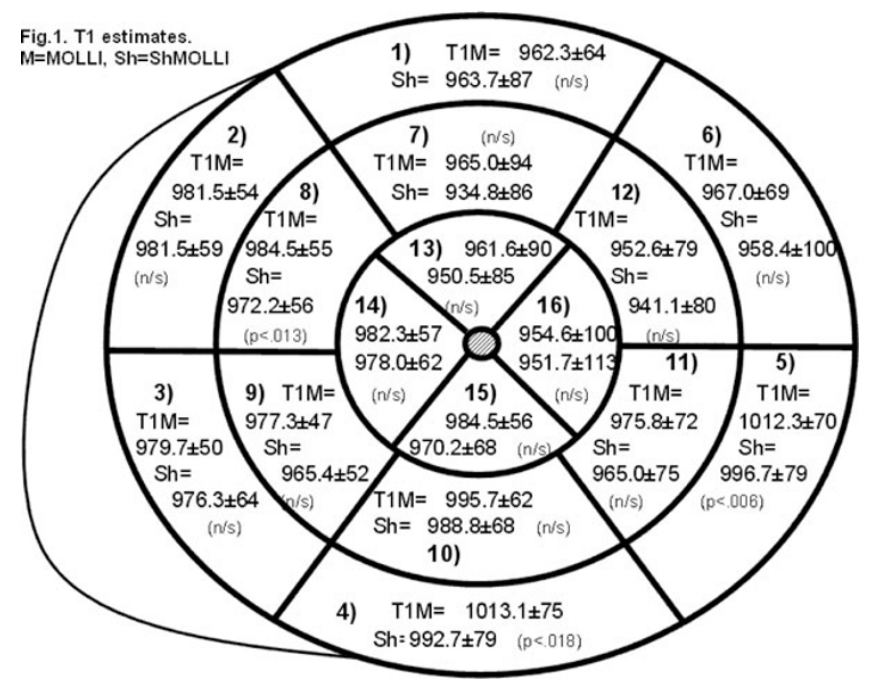

Figure I

TI estimatees. 


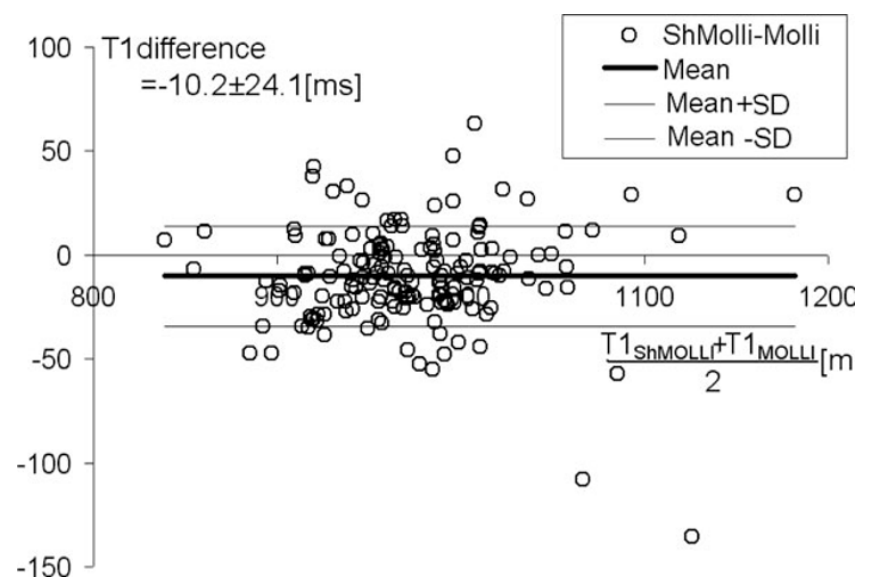

Figure 2

Bland-Altmand plos. TI:ShMOLLI-MOLLI

0.001 , see Fig 2). Sh-MOLLI showed an $8 \%$ larger variation in T1-estimates.

\section{Discussion}

The main advantage of Sh-MOLLI is a near two-fold increase in speed of acquisition. The additional variation in T1-estimates is much less than the $25-50 \%$ expected from the reduced number of samples - likely due to the fact that Sh-MOLLI is less susceptible to movement artifacts accumulated within shorter breatholds. Further underestimation of $\mathrm{T} 1$ values is only minor, especially when compared to the known bias of MOLLI, which is in the order of $-5 \%$. Simulations and phantom measurements (not presented here) showed that the bias in ShMOLLI T1 estimates is relatively stable across a wide range of T1 values and independent of heart rate variation.

\section{Conclusion}

The proposed Sh-MOLLI sequence is fast, clinically applicable, and can generate robust, quantitative single breathhold T1 maps of the human myocardium with high resolution.

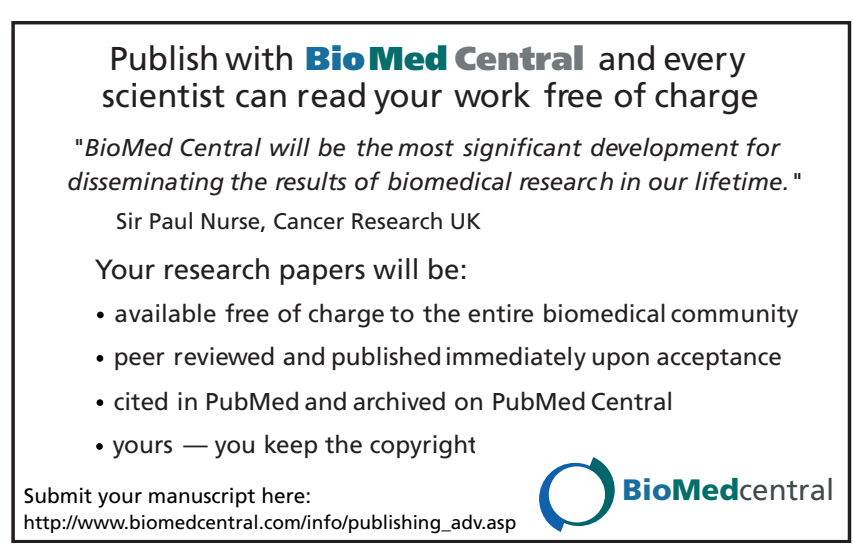

Page 2 of 2

(page number not for citation purposes) 